\title{
Functional differentiation
} CrossMark of 3-ketosteroid $\Delta^{1}$-dehydrogenase isozymes
in Rhodococcus ruber strain Chol-4

\author{
Govinda Guevara', Laura Fernández de las Heras², Julián Perera ${ }^{1}$ and Juana María Navarro Llorens ${ }^{1 *}$ (i)
}

\begin{abstract}
Background: The Rhodococcus ruber strain Chol-4 genome contains at least three putative 3-ketosteroid $\Delta^{1}$ dehydrogenase ORFs (kstD1, kstD2 and kstD3) that code for flavoenzymes involved in the steroid ring degradation. The aim of this work is the functional characterization of these enzymes prior to the developing of different biotechnological applications.

Results: The three $R$. ruber KstD enzymes have different substrate profiles. KstD1 shows preference for 9OHAD and testosterone, followed by progesterone, deoxy corticosterone AD and, finally, 4-BNC, corticosterone and 19OHAD. KstD2 shows maximum preference for progesterone followed by 5a-Tes, DOC, AD testosterone, 4-BNC and lastly 19OHAD, corticosterone and 9OHAD. KstD3 preference is for saturated steroid substrates (5a-Tes) followed by progesterone and DOC. A preliminary attempt to model the catalytic pocket of the KstD proteins revealed some structural differences probably related to their catalytic differences. The expression of $k s t D$ genes has been studied by RT-PCR and RT-qPCR. All the kstD genes are transcribed under all the conditions assayed, although an additional induction in cholesterol and AD could be observed for kstD1 and in cholesterol for kstD3. Co-transcription of some correlative genes could be stated. The transcription initiation signals have been searched, both in silico and in vivo. Putative promoters in the intergenic regions upstream the kstD1, kstD2 and kstD3 genes were identified and probed in an apramycin-promoter-test vector, leading to the functional evidence of those $R$. ruber kstD promoters.
\end{abstract}

Conclusions: At least three putative 3-ketosteroid $\Delta^{1}$-dehydrogenase ORFs (kstD1, kstD2 and kstD3) have been identified and functionally confirmed in R. ruber strain Chol-4. KstD1 and KstD2 display a wide range of substrate preferences regarding to well-known intermediaries of the cholesterol degradation pathway ( $9 O H A D$ and $A D$ ) and other steroid compounds. KstD3 shows a narrower substrate range with a preference for saturated substrates. KstDs differences in their catalytic properties was somehow related to structural differences revealed by a preliminary structural modelling. Transcription of $R$. ruber kstD genes is driven from specific promoters. The three genes are constitutively transcribed, although an additional induction is observed in kstD1 and kstD3. These enzymes have a wide versatility and allow a fine tuning-up of the KstD cellular activity.

Keywords: Rhodococcus ruber, 3-Ketosteroid- $\Delta^{1}$-dehydrogenase, Promoters, Expression, Steroids

\section{Background}

Rhodococci are aerobic Gram-positive soil bacteria belonging to the Actinomycetes group. They show a

\footnotetext{
${ }^{*}$ Correspondence: joana@bio.ucm.es

1 Department of Biochemistry and Molecular Biology I, Universidad Complutense de Madrid, 28040 Madrid, Spain

Full list of author information is available at the end of the article
}

broad catabolic diversity over different substrates, from pollutants to many aromatic compounds, including steroids and sterols [1-3]. Steroids are a source of contamination of soil and waters and their presence has been detected even in drinking water, threatening many ways of life and public health [4-6]. Rhodococci can be useful in this biodegradation field due to their metabolic versatility and steroids degradation capability. On the 
other hand Rhodococcus spp. are potential biotechnological tools $[3,7]$ as they can provide with key enzymes essential for certain reactions that yield industrial needed intermediaries such as 4-androstene-3,17-dione (AD) and 1,4-androstadiene-3,17-dione (ADD) [8].

But before exploiting all the advantages the different rhodococci offer, it is essential to know how these bacteria degrade steroids and which enzymes are involved in this process.

Steroids are molecules with a carbon skeleton of 4 fused rings (A to D) and a side chain up to 10 carbons. During the last years, the increasing number of studies concerning steroid degradation, and more concretely the degradation of cholesterol in bacteria, have clarified some of the catabolic steps (e.g. initiation of the ring degradation by either a $\mathrm{NAD}^{+}$-dependent $3 \beta$-hydroxysteroid dehydrogenase or a cholesterol oxidase) although other steps still remain unclear (e.g. the processing of the $\mathrm{C}$ and $\mathrm{D}$ rings of the steroid structure or the relative order in which the different steps of the degradation of ring and chain occurs) [3, 9-12].

In the general scheme of steroid degradation, there are two key enzymes that initiate the opening of the steroid ring: the 3-ketosteroid- $\Delta^{1}$-dehydrogenase [4-ene-3-oxosteroid: (acceptor)-1-ene-oxoreductase; EC 1.3.99.4)], also known as KstD and the 3-ketosteroid $9 \alpha$-hydroxylase [Androsta-1,4-diene-3,17-dione; EC 1.14.13.142], also known as Ksh [13]. KstD is a flavoenzyme involved in the $\Delta^{1}$-dehydrogenation of the steroid molecule leading to the initiation of the breakdown of the steroid nucleus by introducing a double bond into the A-ring of 3 -ketosteroids $[14,15]$. This flavoprotein converts 4-ene3-oxosteroids (e.g. AD) to 1,4-diene-3-oxosteroids (e.g. ADD) by trans-axial elimination of the $C-1(\alpha)$ and $C-2(\beta)$ hydrogen atoms [16]. KstD homologs have been identified in 100 different bacterial species (78 actinobacteria, 20 proteobacteria and 2 firmicutes) and at least in one fungus, Aspergillus fumigatus CICC 40167 [17, 18]. Most of these KstD-containing bacteria occur in soil, marine or river sediments and are also able to degrade polycyclic aromatic hydrocarbons [19]. Phylogenetic analysis leads to classify the KstD-like enzymes in at least 4 different groups, in which KstD1, KstD2, KstD3 of Rhodococcus erythropolis SQ1 are representatives of three of them [20]. The crystal structure of the enzyme KstD1 of R. erythropolis SQ1 has been elucidated [21] confirming the presence of the two domains previously described, namely a $\mathrm{N}$-terminal flavin adenine dinucleotide (FAD) binding motif and a substrate-binding domain $[14,20,22$, 23].

The substrate range of different KstD proteins has been studied in R. erythropolis SQ1, being 3-ketosteroids with a saturated A-ring (e.g. $5 \alpha$-androstane-3,17-dione and
$5 \alpha$-testosterone) the preferred substrates for KstD3 and (9 $\alpha$-hydroxy-)4-androstene-3,17-dione the favourite one for both KstD1 and KstD2 [20]. It should be mentioned that, apart from their role in steroids degradation, KstD proteins could have specific roles depending of their origin; for instance, the KstD of A. fumigatus CICC 40167 is involved in fusidane antibiotic biosynthesis [17].

We have previously reported the occurrence of three KstD enzymes in R. ruber (NCBI::AFH57399 for KstD1; NCBI::AFH57395 for KstD2 and NCBI::ACS73883 for KstD3) [24]. Growth experiments with single, double or triple $k s t D$ mutants proved that KstD2 is a key enzyme in the transformation of both AD to ADD and 9 $\alpha$-hydroxy4-androstene-3,17-dione (9OHAD) to $9 \alpha$-hydroxy-1,4androstadiene-3,17-dione (9OHADD) while both KstD2 and KstD3 are involved in the cholesterol catabolism in $R$. ruber. On the other hand, the role of KstD1 on the steroids catabolism remains unclear as kstD1 mutation did not affect growing of this strain in steroids [24]. In this study, we cloned the three kstD ORFs and heterologously expressed them in $R$. erythropolis CECT3014, in order to initiate the biochemical characterization of the encoded enzymes, as the basis for further studies on their applications. The results revealed that KstD3 uses more actively substrates with a saturated ring in contrast to KstD1 and KstD2. Additionally, we located and functionally defined the promoters of the three $k s t D$ ORFS in order to provide a basis for future research on the regulation of these genes.

\section{Methods}

\section{Bacterial strains, plasmids and growth conditions}

Rhodococcus ruber strain Chol-4 (CECT 7469; DSM 45280) was isolated from a sewage sludge sample [25]. This strain was routinely grown in Luria-Bertani (LB) or minimal medium (M457 of the DSMZ, Braunschweig, Germany) containing the desired carbon and energy source under aerobic conditions at $30{ }^{\circ} \mathrm{C}$ in a rotary shaker $(250 \mathrm{rpm})$ for 1-3 days. For the steroids growth experiments, a LB pre-grown culture was washed two times with minimal medium prior to inoculation. Cholesterol or AD (Sigma), were added directly to the minimal medium culture for growing and/or induction at 0.6 and $0.44 \mathrm{~g} / \mathrm{L}$, respectively. Escherichia coli strains were grown in LB broth at $37^{\circ} \mathrm{C}, 250 \mathrm{rpm}$. For the promoter growth experiments, cells were plated in minimal medium M457 plates containing the desired carbon source and incubated at $30{ }^{\circ} \mathrm{C}$ for 3 days. Cholesterol and AD were previously dissolved in methyl- $\beta$-cyclodextrin $(C D)$ [26] and prepared as described [27]. Plasmids and bacterial strains used are listed in Additional file 1. Competent cells of $E$. coli DH5 $\alpha \mathrm{F}$ ' and BL21 (DE3) were prepared and transformed by standard protocols [28]. 


\section{Cloning of kstD1, kstD2 and kstD3 of R. ruber strain Chol-4 and heterologous expression in Rhodococcus erythropolis CECT3014 cells}

Chromosomal DNA extraction of $R$. ruber grown in a LB agar plate was performed using the hexadecyltrimethylammonium bromide (CTAB) procedure [29] with the following modifications. Bacterial cells were collected, suspended in $400 \mu \mathrm{L}$ Tris-EDTA buffer $(10 \mathrm{mM}$ Tris/ $\mathrm{HCl}, \mathrm{pH} 8.1 \mathrm{mM}$ EDTA) and incubated at $80{ }^{\circ} \mathrm{C}$ for $20 \mathrm{~min}$. Afterwards, a lysozyme treatment $(50 \mu \mathrm{L}$ of $10 \mathrm{mg} / \mathrm{mL}$ stock) was carried out at $37^{\circ} \mathrm{C}$ for $1-12 \mathrm{~h}$, and then $75 \mu \mathrm{L}$ of SDS containing proteinase $\mathrm{K}(70 \mu \mathrm{L}$ SDS $10 \% \mathrm{wt} / \mathrm{vol}$ plus $5 \mu \mathrm{L}$ proteinase $\mathrm{K} 10 \mathrm{mg} / \mathrm{mL}$ ) was added and incubated for $10 \mathrm{~min}$ at $65^{\circ} \mathrm{C}$. Proteins were precipitated with $100 \mu \mathrm{L}$ of $5 \mathrm{M} \mathrm{NaOH}$ and $100 \mu \mathrm{L} \mathrm{CTAB}(0.1 \mathrm{~g} /$ $\mathrm{mL}$ suspended in $0.7 \mathrm{M} \mathrm{NaOH}$ ) for $10 \mathrm{~min}$ at $65^{\circ} \mathrm{C}$. DNA was purified by extraction with chloroform-isoamyl alcohol (24:1) and phenol-chloroform-isoamyl alcohol (25:24:1) and precipitated with $0.6 \mathrm{vol}$ of isopropanol at room temperature for $30 \mathrm{~min}$. After centrifugation, DNA was washed with $70 \%$ ethanol and suspended in distilled water.

The kstD ORFs were previously identified using the Bioedit program [25] and they were PCR amplified, from start to stop codon, using primers from Additional file 2. PCR was performed under standard conditions using High Fidelity PCR Enzyme Mix (Fermentas) with a specific high GC buffer (Roche) at 30 cycles of $1 \mathrm{~min}$ at $95{ }^{\circ} \mathrm{C}, 1 \mathrm{~min}$ at the desired $\mathrm{Tm}$ and $0.5-3 \mathrm{~min}$ at $72{ }^{\circ} \mathrm{C}$ (unless stated otherwise).

NdeI-BamHI (kstD3), NdeI-BglII (kstD2 and kstD1) restricted PCR products were first cloned into pGEM-T Easy Vector (Promega) and then moved to the shuttle vector pTip-QC1 [30]. Expression plasmids either with or without a kstD ORF were used to electroporate Rhodococcus erythropolis CECT3014. Selection was made in LB with $34 \mu \mathrm{g} / \mathrm{mL} \mathrm{Cm}$. Cultures of $R$. erythropolis harbouring expression plasmids (pTip-KsTD1, pTipKstD2, pTip-KstD3 or pTip-QC1) were grown $\left(30{ }^{\circ} \mathrm{C}\right.$, $200 \mathrm{rpm}$ ) in $50 \mathrm{~mL}$ LB broth supplemented with $\mathrm{Cm}$ until an $\mathrm{OD}_{600 \mathrm{~nm}}$ of 0.6-0.8. After that, expression was induced by adding $1 \mu \mathrm{g} / \mathrm{mL}$ thiostrepton (Sigma) to the culture. Cells were kept growing for $24 \mathrm{~h}$ more and they were collected at $5000 \mathrm{rpm}$ for $10 \mathrm{~min}$, washed twice in $50 \mathrm{mM}$ phosphate buffer $\mathrm{pH} 7.0$, concentrated to $5 \mathrm{~mL}$ and sonicated. The resulting cell extracts were used for analysis of KstD activity with a range of steroid substrates. Total protein content was measured by Bradford assay [31]. Samples were analysed by PAGE-SDS in $12.5 \% \mathrm{wt} / \mathrm{vol}$ gels and using $10 \mu \mathrm{g}$ of total protein per lane.

\section{KstD enzymatic assay}

The kinetic of the enzymatic extracts was determined as previously stated $[20,32]$. The cell-free extracts were incubated with AD (Sigma), 9OHAD (Organon Biosciences), $17 \beta$-hydroxy- $5 \alpha$-androstan-3-one or $5 \alpha$-Tes, DOC (deoxycorticosterone), testosterone, corticosterone, 19OHAD, progesterone (Sigma), 4-BNC (4-pregnen-3-one-20 $\beta$ carboxylic acid) (Steraloids), 5 $\beta$-androstane-3,17-dione (Steraloids), 4-cholestene-3-one (Sigma) or $5 \alpha$-cholestan3-one (Acros Organics). Structures of steroids used in this study are shown in Additional file 3. Enzyme activities were measured spectrophotometrically at $30^{\circ} \mathrm{C}$ using 2,6-dichlorophenol-indophenol (DCPIP, Sigma) as an artificial electron acceptor. The reaction mixture $(1 \mathrm{~mL})$ consisted of $50 \mathrm{mM}$ Tris (pH 7), $80 \mu \mathrm{M}$ DCPIP, cell-free extract and $200 \mu \mathrm{M}$ steroid in ethanol (methanol in the case of $5 \alpha$-cholestane-3-one). Four replicates were analysed. Activities are expressed as mean values $\pm \mathrm{SD}$ in units per milligram of protein; one unit is defined as the amount of enzyme which causes the reduction of $1 \mu \mathrm{mol}$ of DCPIP/min $\left(\varepsilon_{600}=21 \mathrm{mM}^{-1} \mathrm{~cm}^{-1}\right)$ after taking into account the value of the activity of control (cells harbouring the empty pTip-QC1 vector) for a certain steroid. Total protein concentration $(\mathrm{mg} / \mathrm{mL})$ was measured by Bradford assay [31]. The kinetics of the KstD enzymes were determined by incubating the cell-free extracts with varying concentrations of steroid substrates. The kinetics parameters were analysed by nonlinear regression curve fitting of the data to the Michaelis- Menten equation using Hyper32 1.0 software (Informer Technologies, http://hyper32.software.informer.com/).

\section{Expression analysis by RT-PCR and RT-qPCR}

RNA samples for RT-PCR experiments were obtained from mid-log exponential phase cultures $\left(\mathrm{OD}_{600 \mathrm{~nm}} 0.7-\right.$ $0.8)$. Total RNA was prepared with the RNeasy Mini Kit (Qiagen) following the manufacturer's indications with the following modification: $50 \mathrm{mg}$ of acid-washed glass beads ( $150 \mu \mathrm{m}$ diameter) were added in the first step and each sample was shaken at maximum speed in a Bullet Blender for $5 \mathrm{~min}$. The cell debris was removed by centrifugation. The supernatant was subjected to the RNeasy Mini Kit (Qiagen) protocol. The total RNA obtained $(0.5-1 \mu \mathrm{g})$ was treated once with $5 \mathrm{U}$ of Turbo DNase RNase-Free (Ambion) in a $700 \mu \mathrm{L}$ volume for $2 \mathrm{~h}$ at $37^{\circ} \mathrm{C}$. RNA samples were extracted with 1 volume of acid phenol (Sigma), vigorously shaken and incubated at room temperature for $15 \mathrm{~min}$. After $15 \mathrm{~min}$ centrifugation, the upper phase was precipitated by addition of 0.12 volumes of $5 \mathrm{M} \mathrm{NH}_{4} \mathrm{Ac}, 0.02$ volumes of glycogen $(5 \mathrm{mg} / \mathrm{mL})$ and 1 volume of isopropanol, washed twice in $70 \%$ ethanol 
and dissolved in water. Samples were treated with DNase until no DNA was detected by PCR to avoid DNA contamination. The RNA concentration was then evaluated using a NanoDrop Spectrophotometer ND-1000.

For the RT-PCR the cDNA was synthesized using SuperScrip III Reverse Transcriptase (Invitrogen) following the manufacturer's indications. cDNA was used as template (25 ng) for PCR reactions ( $20 \mu \mathrm{L}$ final volume). Controls without reverse transcriptase (RT-) were used to detect any contamination of undigested DNA in the RNA preparations. PCR products were analysed in $0.8 \%$ agarose gels.

To quantify the expression of the three KstD genes, a RT-qPCR analysis was performed using RNA from wildtype strains cultured in M457 minimal medium containing the desired carbon and energy source $(2 \mathrm{~g} / \mathrm{L}$ sodium acetate, $0.44 \mathrm{~g} / \mathrm{L} \mathrm{AD}$ or $0.6 \mathrm{~g} / \mathrm{L}$ cholesterol). The RNA quality was assessed by using Bioanalyzer 2100 (Agilent). cDNA was synthetized using $1 \mu \mathrm{g}$ of RNA with the high capacity RNA to cDNA Kit (Applied Biosystems). The RT-qPCR analysis of cDNA was performed on Applied Biosystems QuantStudio 12K Flex Real-Time PCR Systems. The reaction conditions were $10 \mathrm{~min}$ at $95{ }^{\circ} \mathrm{C}$ followed by 40 cycles of $15 \mathrm{~s}$ at $95{ }^{\circ} \mathrm{C}$ and $1 \mathrm{~min}$ at $60{ }^{\circ} \mathrm{C}$ for extension. The temperature of the melting curve was from 60 to $95{ }^{\circ} \mathrm{C}$. The FAD-binding dehydrogenase D092_14375 gene was used as an internal control to normalize messenger RNA levels. All reactions were performed in triplicate. The RT-qPCR experiment and the analysis of the relative fold difference of each gene using the $2^{-\Delta \Delta \mathrm{Ct}}$ algorithm was performed in the Genomic unit of Universidad Complutense de Madrid.

The sodium acetate grown culture was used as the reference medium. Therefore, the relative expression indicates how many times the expression level of a certain gene is detected respect to the levels detected when growing on sodium acetate.

\section{In silico analyses}

DNASTAR (Lasergene) programs were used to analyse sequences and to design primers. The $R$. ruber strain Chol-4 genomic DNA has been previously sequenced [33]. BioEdit program was used to perform local-blast alignments within the genome data (NCIB::ANGC01000000). Putative signal peptides were predicted by SignalP 4.1 server using a model trained on Gram-positive bacteria [34]. Sigma 70 putative promoters predictions were performed using the BPROM server, a bacterial sigma 70 promoter recognition program with about $80 \%$ accuracy and specificity [35], the Neural Network Promoter Prediction (NNPP) based on prokaryotes [36] in all cases with a score value of $\geq 80 \%$, or the webserver PePPER for prediction of prokaryote promoter elements and regulons [37].
For the protein modelling we employed different software. I-Tasser (http://zhanglab.ccmb.med.umich.edu/ITASSER) was used for an approach to protein structure and function prediction [38] and PredictProtein (www. predictprotein.org/home) was used for the secondary structure, solvent accessibility and transmembrane helix prediction [39]. COBALT was used as a multiple sequence alignment tool to find similarities among the catalytic residues (www.ncbi.nlm.nih.gov/tools/cobalt/ re_cobalt.cgi) [40] and PyMOL (www.pymol.org/) as a molecular visualization system (PyMOL Molecular Graphics System, Version 0.99, Schrödinger).

\section{Promoter cloning and characterization}

The NheI-Pci $0.4 \mathrm{~Kb}$ multiple cloning site (mcs) from pSEVA351 [41, 42] was cloned into pNV119 vector [43], from now on named as pNVS (Additional file 4).

The putative $k s t D$ promoter sequences were amplified by PCR, from the end of the upstream flanking gene to the end of the first six amino acid codifying sequence. The $X b a \mathrm{I}, P s t \mathrm{I}$-flanked $k s t D$ promoter regions (kstD1p, $k s t D 2 p$ and $k s t D 3 p)$ and the KpnI, PstI-flanked $k s t D 3^{b} p$ minimal promoter were cloned into pNVS. The resulting vectors were designated pNVSP1, pNVSP2, pNVSP3 and pNVSP3 $^{\mathrm{b}}$, respectively.

Apramycin resistance gene $\left(A m^{r}\right)$ was amplified by PCR from plasmid pIJ773 [44], from start to stop codon. The NruI/HindII digested $\mathrm{Am}^{\mathrm{r}}$ fragment was cloned in each one of the previous $k s t D$ constructions. The resulting plasmids were checked by sequencing (Secugen) and named pNVSP1-A, pNVSP2-A, pNVSP3-A and pNVSP3 ${ }^{\mathrm{b}}-\mathrm{A}$, respectively.

All the primers used are listed in Additional file 2. PCR was performed under standard conditions using High Fidelity PCR (Roche) with glycerol 5\% and a basic program unless stated otherwise.

As a control, a plasmid without any promoter but carrying the $\mathrm{Am}^{\mathrm{r}}$ gene (pNVSA) was made by digesting pNVSP1-A with NruI-XbaI; the resulting $4.2 \mathrm{~Kb}$ fragment was cut from a $1 \%$ agarose gel and purified with GENECLEAN Turbo Kit. Blunt ends suitable for ligation with T4 DNA ligase (Takara) were generated using the End repair kit (DNA terminator, Lucigen). The final ligated product was used to transform E. coli $\mathrm{DH} 5 \alpha \mathrm{F}^{\prime}$. Deletion was checked by sequencing (Secugen).

Every one of the plasmid set was introduced in $R$. ruber strain Chol-4 by electroporation (200 or $400 \mu \mathrm{L}$ cells with $1 \mu \mathrm{g}$ DNA at $400 \Omega, 25 \mathrm{~mA}, 2.5 \mu \mathrm{F} ; 10-11 \mathrm{~ms})$, the resulting cells were suspended in $800 \mu \mathrm{L}$ of $\mathrm{LB}$ and kept for 6 min at $46{ }^{\circ} \mathrm{C}$, and then for $5 \mathrm{~h}$ at $30^{\circ} \mathrm{C}$ without shaking. Finally, they were plated on LB Agar with $200 \mu \mathrm{g} / \mathrm{mL}$ kanamycin and kept at $30{ }^{\circ} \mathrm{C}$. To verify the presence of plasmids, two colonies of each plate were 
picked and grown in $3 \mathrm{~mL}$ of LB-200 $\mu \mathrm{g} / \mathrm{mL}$ kanamycin. Plasmid were extracted using the method described in Hopwood et al. [45] and used to transform E. coli strain DH5 $\alpha F^{\prime}$ [28]. Plasmids obtained from E. coli colonies grown at $37^{\circ} \mathrm{C}$ in $50 \mu \mathrm{g} / \mathrm{mL}$ kanamycin were verified by sequencing (Secugen). Finally, those colonies of $R$. ruber strain Chol-4 harbouring the right recombinant plasmids were picked and grown in agar minimal media at $30{ }^{\circ} \mathrm{C}$ with different carbon source with or without apramycin $(300 \mu \mathrm{g} / \mathrm{mL})$ or kanamycin $(200 \mu \mathrm{g} / \mathrm{mL})$.

To define the transcriptional start sites (TSS) of kstD promoters, a transcription start point protocol (ARFTSS) [46] was used on $R$. ruber cells with the following modifications.

RNA from $R$. ruber cells growing in different carbons sources cultures was isolated. The culture media were: LB for kstD1 TSS, minimal medium supplemented with AD for kstD2 TSS and minimal medium supplemented with cholesterol for kstD3 TSS. Total RNA was isolated as described previously [47]. It was qualified by electrophoresis and quantified by Nanodrop 1000 (NanoDrop Technologies). $20 \mu \mathrm{g}$ of RNA were reverse transcribed with a gene specific phosphorylated $5^{\prime}$-end primer ( $\mathrm{R} 1$ for each $k s t D$ ) using SuperScrip III Reverse Transcriptase from Invitrogen. As a result, cDNA fragments with the TSS as $3^{\prime}$-end were generated and purified. After removing of RNA with $0.5 \mu \mathrm{g} / \mu \mathrm{L}$ RNase A $\left(37^{\circ} \mathrm{C}\right.$ for $\left.30 \mathrm{~min}\right)$, cDNA was purified using the GENECLEAN turbo Kit (MPI) and final products were treated with T4 RNA ligase from Fermentas (10U, $37^{\circ} \mathrm{C}$ for $30 \mathrm{~min}$ ). This T4 RNA ligase catalyses the ATP-dependent intra- and intermolecular formation of phosphodiester bonds between $5^{\prime}$-phosphate and $3^{\prime}$-hydroxyl termini of oligonucleotides, single-stranded RNA and DNA. It was used in this study to circularize the cDNA. The circularized cDNAs were then used as template for PCR amplification with Expand High Fidelity (Roche) using R2 and F3 primers specific for each $k s t D$ ORF. PCR products were purified using the GENECLEAN turbo Kit and sequenced (Secugen). The nucleotide upstream the $5^{\prime}$-end of the R1 primer is the transcription initiation site.

\section{Results and discussion}

As we have published earlier, the $R$. ruber strain Chol-4 genome contains three putative 3-ketosteroid $\Delta^{1}$ dehydrogenase ORFs (kstD1, kstD2 and kstD3) that code for flavoenzymes involved in the steroid ring degradation [24]. Growth experiments with $k s t D$ mutants proved that KstD2 is the main enzyme involved in the transformation of AD to ADD. R. ruber kstD2 mutants accumulate 9OHAD from AD due to the action of a 3-ketosteroid$9 \alpha$-hydroxylase (KshAB). On the other hand, only the strains lacking both KstD2 and KstD3 were unable to grow in minimal medium with cholesterol as the only carbon source. In order to know more about these $R$. ruber enzymes, we have performed transcriptional studies and followed their heterologous expression in $R$. erythropolis to detect their activities on a set of different substrates.

\section{In silico analyses of $k s t D$ promoter regions of $R$. ruber strain Chol-4}

A scheme of the three $R$. ruber $k s t D$ ORFs and their genomic surroundings is depicted in Fig. 1 showing also the in silico predicted $\mathrm{pP} 1, \mathrm{pP} 2, \mathrm{pP} 3, \mathrm{pP} 4$ and $\mathrm{pP} 5$ promoters. The available programs (see "Methods") yielded no putative promoter region just upstream either kstD1 or kstD2 ORFs, although it should be noted here that promoter prediction programs are not specific for Grampositive species.

However, these programs detected putative promoters (pP1, pP2 and pP4) upstream the cyp 450 gene, lying in the intergenic region of the fadA5-hyp and cyp450kstD1-MFSt opposite clusters. Flanking the pP1 putative promoter there are two palindromic sequences (TagAACagGTTgtc and TagAACgtGTTccA) (Fig. 2), one of them rather similar and the second one identical to the consensus binding region reported for the KstR regulatory protein of Mycobacterium (TnnAACnnGTTnnA) [48]. KstR and KstR2, two TetR family repressor regulators, have been found to control most of the steroid pathways in actinobacteria [48-50]. KstR is a highly conserved Tet $R$

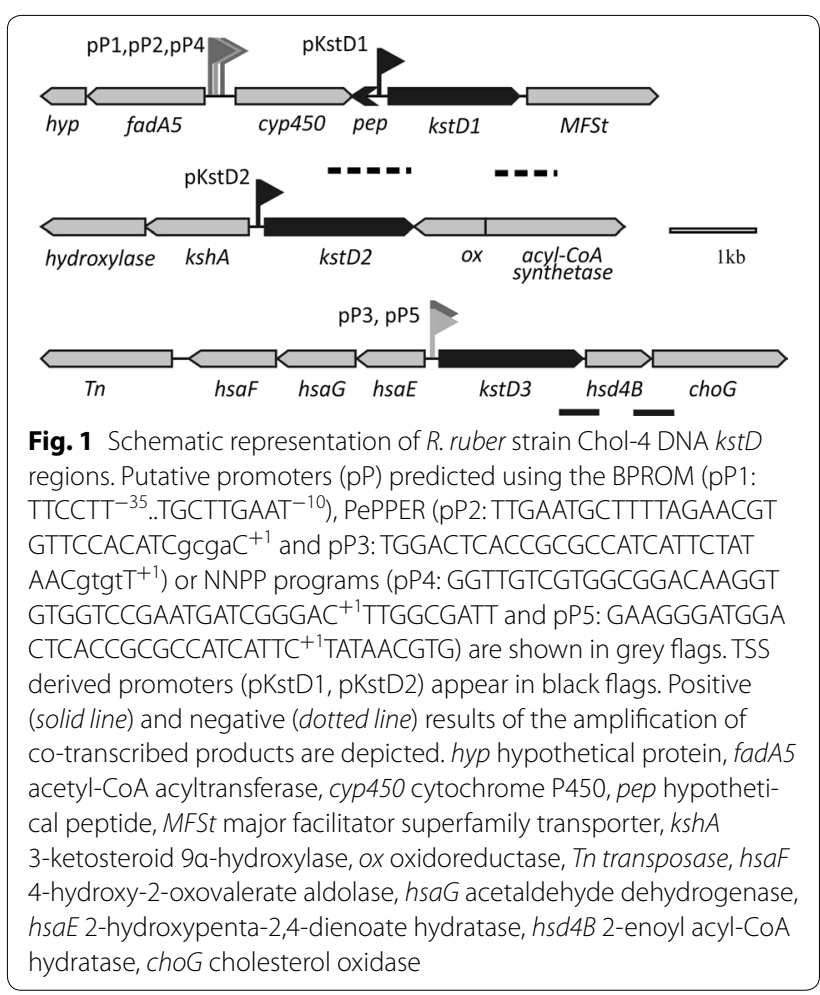



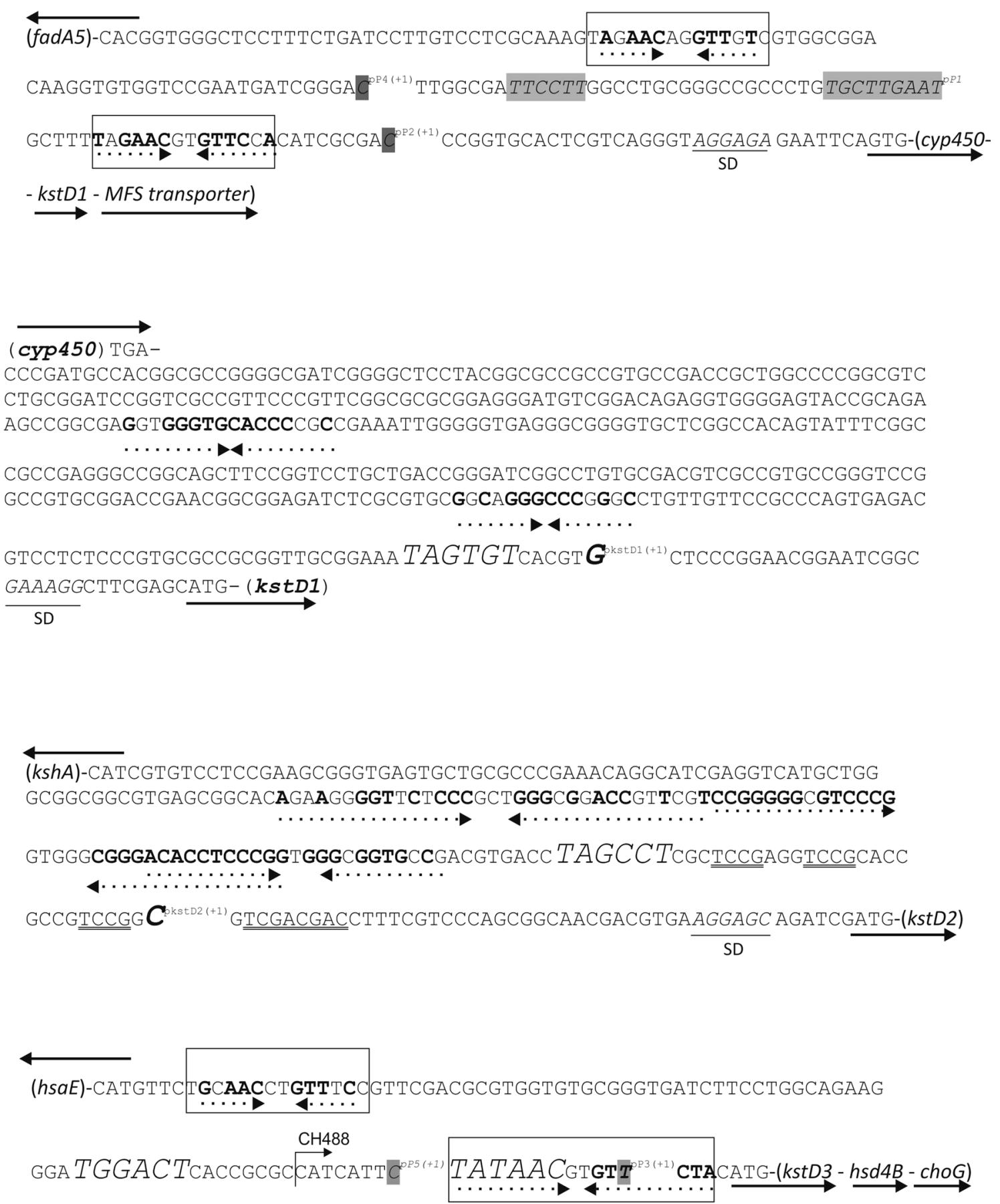

Fig. 2 Sequence of regions upstream kstD ORFs. Solid arrows represent the orientation of the different ORFs from the initiation to the final codon. Sequences similar to Mycobacterium KstR binding sites (TnnAACnnGTTnnA) are within a square. Palindromic sequences appear in bold characters and dotted underlined. Shine Dalgarno (SD) sequences are in italics and underlined. The $-10,-35$ boxes are marked in grey. The in silico transcription initiation point of the putative $\mathrm{PP} 1, \mathrm{pP} 2, \mathrm{pP} 3$ and $\mathrm{pP} 4$ promoters are in italics and marked as $\mathrm{pP}(+1)$. The transcription start sites of kstD1 and kstD2 ORF obtained by the ARF-TSS method are shown in bold italics and marked as pKstD(+1). The TCCG repeats and the Sal box upstream kstD2 are double underlined. Promoter signals similar to others described (e.g. M. tuberculosis) [55] appear in bigger size. Primer CH488 indicating the beginning of the minimum kstD3 promoter is also shown 
family repressor that regulates the transcription of genes related to the upper and central pathway of cholesterol catabolism, namely the membrane transport of cholesterol, the degradation of the steroid side chain and the opening of the A and $B$ rings $[12,48]$. Upon binding to a 3-oxo-4-cholestenoic acid or to a CoA thioester cholesterol metabolite, KstR releases the DNA and allows transcription to begin $[51,52]$. The KstR binding motif has been proved to be conserved within actinobacteria [48, 53].

The possible co-transcription of the cyp450-kstD1MSFt ORF cluster to a polycistronic mRNA would come into contradiction with the transcription of a non-yet described short putative ORF (pep, Fig. 1) located in opposite sense in the 425 bp cyp450-kstD1 intergenic region. This short ORF might code for a 35 amino acid peptide which shows a $98 \%$ amino acid identity with part of the hypothetical 78 aa protein RHRU231_750039 ( $R$. ruber, 78 aa). Therefore, $k s t D 1$ might be independently transcribed while $\mathrm{pP} 1 / \mathrm{pP} 2 / \mathrm{pP} 4$ putative promoters might be only involved in cyp 450 transcription. Moreover, a putative ribosome binding site (GAAAGG) was found 9 bp upstream the kstD1 initiation codon (Fig. 2) that is identical to the proposed one for sigA of $R$. ruber $\mathrm{TH}$ [54].

In the case of the kstD2 ORF, none of the online programs recognized a promoter consensus, although this region contains some quasi-palindromic sequences and a Shine-Dalgarno-like motif (AGGAGC) (Fig. 2).

There are two putative promoters for the kstD3 ORF (pP3 and pP5, see Figs. 1 and 2) that lie in the intergenic region between $h s a E$ and $k s t D 3$ ORFs. The putative promoter pP3 contains the sequence TATAAC similar to the -10 consensus motif described for $M$. smegmatis promoters ( $\left.\mathrm{T}_{100 \%} \mathrm{~A}_{93 \%} \mathrm{~T}_{50 \%} \mathrm{~A}_{57 \%} \mathrm{~A}_{43 \%} \mathrm{~T}_{71 \%}\right)$ [55], and a -35 region (TGGACT) that resembles the E. coli promoter consensus motif TTGACA. In this region, there are also a tandem of two putative KstR binding sequences around this promoter (TgcAACctGTTtcc and TatAACgtGTTctA), one quite similar and the other identical to the KstR binding consensus, in a similar way to the cyp 450 pP1/pP2 putative promoter (Fig. 2). The arrangement of these promoter and regulatory sequences, lying between opposite cluster genes, also occurs in the $R$. ruber kstD1 region and it is similar to that found in Mycobacterium and Rhodococcus jostii RHA1 genomes [48, 56]. On the other hand, Shell et al. have recently described that the abundance of leaderless transcripts (that lack a $5^{\prime}$ UTR and a Shine-Dalgarno sequence and that begin with ATG or GTG) is a major feature of mycobacterial that accounts for around one-quarter of the transcripts [57]. $k s t D 3$ could be a leaderless ORF: there is no evidence of a Shine-Dalgarno sequence in its $5^{\prime}$ region and the putative promoter is quite near to the ATG initiation codon. Moreover, as it will be stated later, the promoter of this intergenic region is functional in Rhodococcus but not in $E$. coli, a fact that has also been confirmed in the mycobacterial leaderless messenger translation [57].

\section{Promoter cloning and characterization}

To go further in the characterization of the promoter regions of the $R$. ruber kstD ORFs, a promoter-test vector suitable for $R$. ruber strain Chol-4 was constructed. $R$. ruber is sensitive to apramycin so we chose the expression of a gene encoding this resistance as a proof of the promoter activity.

pNV119 [43], a Nocardian shuttle vector shown to replicate in $R$. ruber [24], was modified by adding the mcs of pSEVA351 that contains a transcriptional terminator in each extreme [41, 42]. The resulting pNVS plasmid (see Additional file 4) was used to study the activity of the Chol-4 putative promoter regions. The three $k s t D$ intergenic regions (Fig. 2) plus the first 21 bases of each $k s t D$ ORF were PCR amplified, transcriptionally fused to the apramycin resistance gene obtained from pIJ773 and cloned into the mcs of pNVS. The recombinant plasmids were introduced into $R$. ruber by electroporation and kanamycin resistant clones were selected. $R$. ruber clones, harbouring the plasmids pNVSP1-A, pNVSP2-A or pNVSP3-A, were then plated in minimal medium supplemented with either $1.5 \mathrm{mM}$ cholesterol, $1.5 \mathrm{mM} \mathrm{AD}$ or $10 \mathrm{mM}$ sodium acetate, and in the presence of either $200 \mu \mathrm{g} / \mathrm{mL}$ kanamycin or $300 \mu \mathrm{g} / \mathrm{mL}$ apramycin. The apramycin resistance gene $\left(\mathrm{Am}^{\mathrm{r}}\right)$ without any upstream promoter region was cloned in pNVS mcs generating the vector pNVSA that was used as a negative control. As a second control, a set of pNVSP vectors that contain every promoter region but do not carry the apramycin resistance gene was used. Figure 3 shows that only the cells harbouring the double system formed by a putative promoter and the apramycin resistance gene were able to grow on apramycin and kanamycin while cells harbouring the pNVSA or the pNVSPs vector were only able to grow in kanamycin plates. These results unambiguously confirm that all the three checked DNA regions contain $R$. ruber promoter sequences functionally active in the conditions used. On the other hand, E. coli harbouring the plasmids pNVSP1-A or pNVSP2-A were also able to grow in apramycin, in contrast with those harbouring pNVSP3-A (data not shown). Other actinobacteria promoters (e.g. some of Mycobacterium and Streptomyces spp.; $[55,58]$ are also not functional in $E$. coli strains; this fact could related to the occurrence of leaderless genes [57].

Although none of the online programs recognized a promoter consensus for the $k s t D 2 \mathrm{ORF}$, the promoter-less 


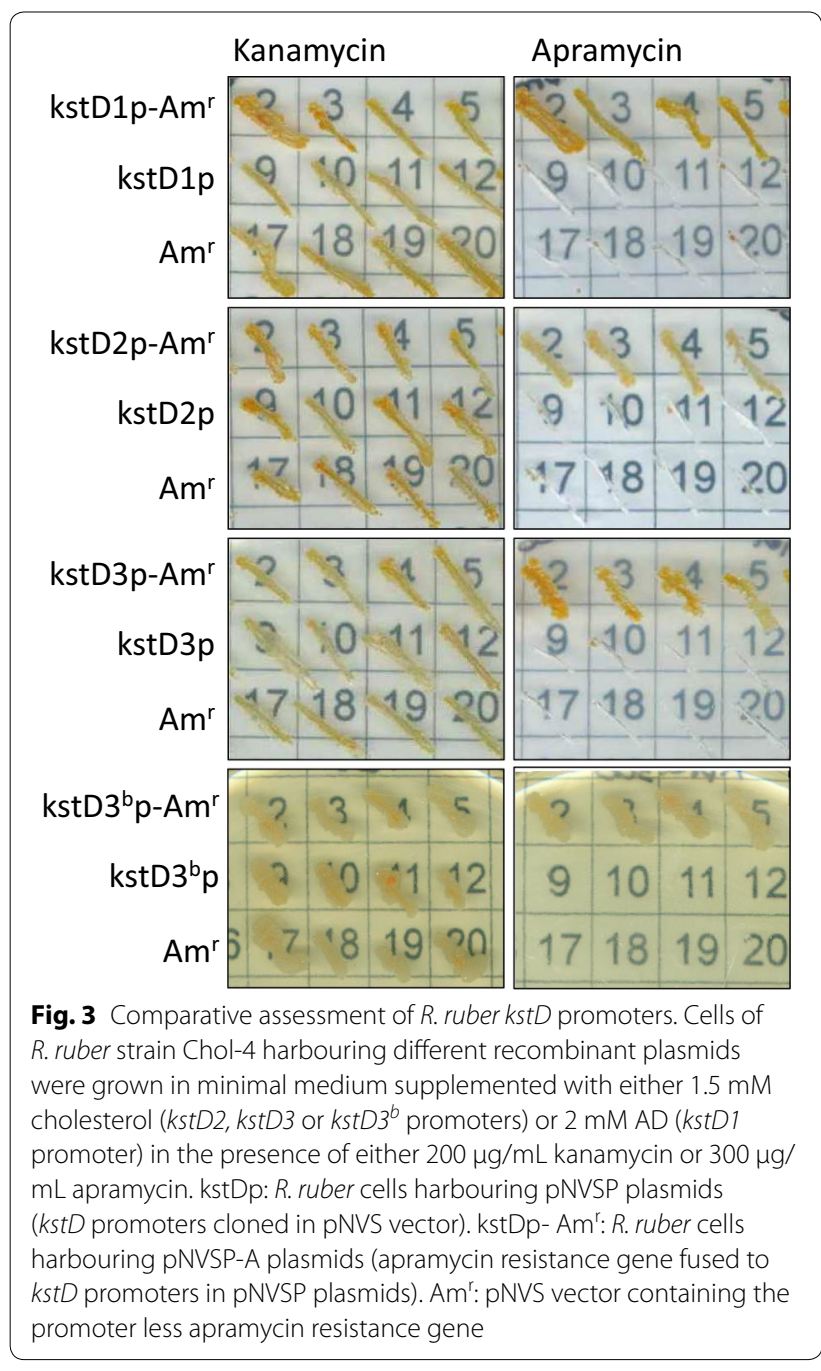

vector pNVS has enabled us to check the promoter activity of the intergenic regions. The construction of an improved version of this plasmid that allows a quantitative analysis of promoter strength is under work.

To define the transcription start sites (TSS) of the $k s t D$ genes, the transcription start point protocol (ARFTSS) indicated in "Methods" section was followed in $R$. ruber cells. We could conclude that the $5^{\prime}$ terminal base in $k s t D 1$ messenger RNA is a G resulting from the transcription that starts $34 \mathrm{bp}$ upstream the kstD1 initiation codon. Similarly, the transcription start site of the $k s t D 2$ gene is a $C 48$ bp upstream the kstD2 initiation codon (Figs. 2, 4). However, this approach did not yield any result in the case of the kstD3 gene. In order to better define the limits of the $k s t D 3$ promoter, progressively shorter sections of the intergenic region, keeping the first 21 bases of kstD3 ORF, were PCR amplified and transcriptionally fused to the apramycin resistance gene. As it can be seen in Figs. 2 and 3, just a minimum region of

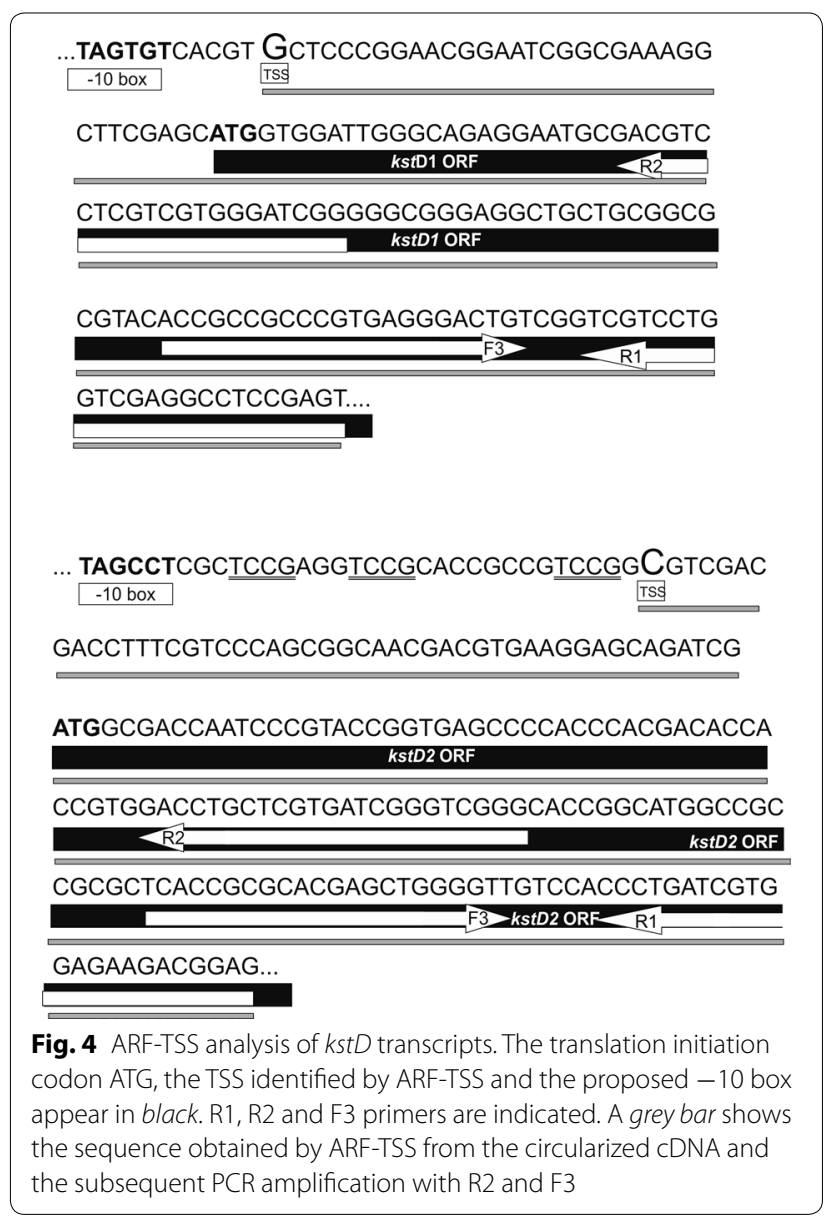

only 23 pb upstream the ATG of kstD3 ORF (pNVSP3 ${ }^{\text {b }}$ A vector) was enough to act as a promoter as the Rhodococcus cells harbouring this vector were able to grow on medium with either apramycin or kanamycin. This promoter region is partially similar to the putative pP3 and $\mathrm{pP} 5$ promoters mentioned before, containing the TATAAC sequence similar to the -10 consensus motif described for Mycobacterium smegmatis promoters (Fig. 1).

Putative -35 and -10 hexamers identification was based on the sequence of other actinobacteria promoters. The -10 region motif (TAGTGT) found 40 bases upstream the $k s t D 1$ initiation codon is similar to the -10 consensus region described for Mycobacterium tuberculosis promoters $\left(\mathrm{T}_{80 \%} \mathrm{~A}_{90 \%} \mathrm{Y}_{60 \%} \mathrm{G}_{40 \%} \mathrm{~A}_{60 \%} \mathrm{~T}_{100 \%}\right)$ and identical to the T101 promoter described by Bashyam et al. in the same bacteria [55]. No sequence similar to any -35 motif was found in the kstD1 region. The absence of -35 motifs seems to be a characteristic of actinobacterial genes $[55,58]$.

There is a putative -10 sequence (TAGCCT) in the intergenic region between $k s h A$ and $k s t D 2$ that resembles 
the T6 promoter of $M$. tuberculosis (TAGGCT) [55]. However, it is a bit far away from the kstD2 TSS. There is a repetitive motif TCCG in this region (Fig. 2) that also appears in the human Sal box element present in the $3^{\prime}$ terminal spacer of rDNA and that constitutes a termination signal for RNA polymerase I (TCCGCACGGGTCGACCAG) [59]. In this kstD2 upstream region we found something similar: TCCGAGGTCCGCACCGCCGTCCGGCGTCGACGAC, where black-underlined letters marked the resemblance between them. Such promoter proximal terminators can appear also upstream of the transcription start site and in this case, they are described to positively affect transcription initiation and to prevent transcriptional interference by reading through of polymerases from the spacer that separates each rDNA

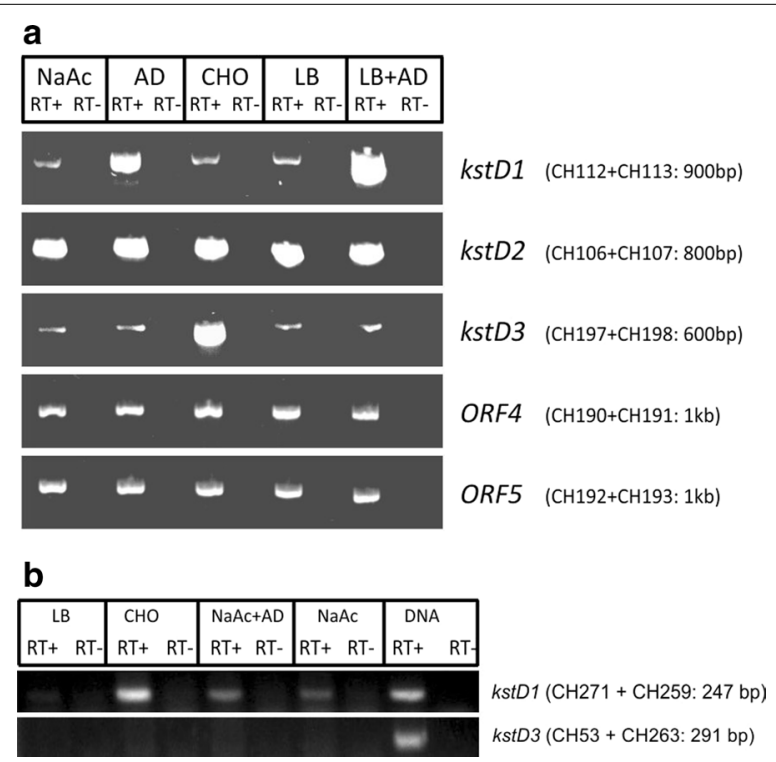

Fig. 5 a Analysis of transcription products of kstD genes in $R$. ruber strain Chol-4 by specific RT-PCR amplification and agarose gel electrophoresis. RNA samples were isolated from cultures grown in: M457 minimal medium supplemented with sodium acetate (lane $\mathrm{NaAc}$ ), AD (lane $\mathrm{AD}$ ) or cholesterol (lane $\mathrm{CHO}$ ) and LB medium supplemented with (lane $L B+A D$ ) or without $A D$ (lane LB). RT—refers to negative controls (not incubated with retrotranscriptase) to exclude DNA contamination in RNA samples. Primers used in each PCR reaction and amplified fragment size are shown in brackets. As non-induced expression controls, the transcription of two R. ruber Chol-4 enzyme genes was followed in the same conditions: ORF4, that codes for a FAD-binding dehydrogenase D092_14375 (99\% identity with the protein NCIB::WP_010594120.1 of Rhodococcus sp. P14) and ORF5 (D092_13875) that codes for a fumarate reductase (99\% identity with NCIB::WP_010594021.1 of Rhodococcus sp. P14; see sequence in Additional file 6). the FAD-binding dehydrogenase D092_14375 and the fumarate reductase D092_13875. b RT-PCR assays of $R$. ruber Chol-4 kstD1 and kstD3 transcription carried out in kstD2 R. ruber mutant growing in: LB (lane LB) or M457 plus CHO (lane CHO), NaAc and AD (lane NaAc $+\mathrm{AD}$ ) or NaAc (lane NaAc). Lane DNA shows the result of the control experiment: PCR made on $R$. ruber DNA repetition [60-62]. The biological significance of this motif in this intergenic kstD2 region should be further determined.

\section{Transcriptional analysis of $k s t D$ genes in $R$. ruber strain Chol-4}

The transcription of the three $k s t D$ genes found in $R$. ruber strain Chol-4 was analysed by RT-PCR of RNA samples prepared from cultures grown in either M457 mineral medium or LB medium supplemented with either AD or cholesterol as possible inductors.

Control cultures were grown in either M457 supplemented with sodium acetate $(2 \mathrm{~g} / \mathrm{L} \mathrm{NaAc})$ or $\mathrm{LB}$, both in the absence of any steroid. Using the specific primer pairs designed to search for the transcript of each different ORF (Additional file 2), we could show that all the $k s t D$ genes are transcribed in all the conditions used in our assays (Fig. 5) and that transcription of some of them is induced by cholesterol or AD. There are also other Rhodococcus metabolic genes reported to show a low-level constitutive transcription that can be strongly induced under the presence of a determinate substrate. For instance, phenol degradation genes in $R$. erythropolis are constitutively transcribed and also highly induced by phenol [63].

cDNA templates concentrations were adjusted to the same value in RT-PCR experiments, so we can consider the differences observed in the thickness of some amplification bands to be meaningful (Fig. 5). Specific amplification of kstD1 cDNA was higher in AD induced than in non-induced cultures, while amplification of kstD3 cDNA was higher in cultures induced with cholesterol as compared to the other conditions assayed. So we can conclude that these two genes, although constitutively transcribed, they can also be additionally induced by the presence of $\mathrm{AD}(k s t D 1)$ and cholesterol (kstD3). The induction of $k s t D$ genes by cholesterol or $\mathrm{AD}$ has also been reported in other microorganisms: a 3.3 expression ratio (cholesterol/pyruvate) was reported for one kstD in $R$. jostii RHA1 (ro04532) [64, 65], while other putative $k s t D$ genes of the same strain (e.g. ro02483, ro05798 and ro05813) were up-regulated in 7-ketocholesterol but not in cholesterol [64]; the main kstD in M. smegmatis (MSMEG_5941) was 13-fold up-regulated in cholesterol respect to glycerol [12]; a 1.8, 4.1 or 1.2 -fold up-regulation (AD/glycerol) was found for $k s t D 1, k s t D 3$ and $k s t D 2$ genes respectively in Mycobacterium neoaurum [66]. These differences in regulation among the different $k s t D$ genes within the same strain highlight the view that KstD proteins may be acting in different metabolic steps and/ or pathways, each one having a particular catalytic role.

The amplification of $R$. ruber strain Chol-4 kstD2 cDNA yielded thick amplification bands in all cases 
(Fig. 5), which clearly leads to propose a constitutive expression of $k s t D 2$. In a similar way, two putative $k s t D$ genes in $R$. jostii RHA1 genome (ro90203 and ro09040, belonging to the KstD2-branch of the KstD phylogenetic tree) [20] were expressed but not up-regulated neither in cholesterol nor in 7-ketocholesterol, when compared to pyruvate [64].

The expression profile of $R$. ruber kstD1, kstD2 and $k s t D 3$ genes was determined by real-time PCR. Taking as 1 the expression levels on sodium acetate (unexposed steroid culture), the values obtained for the expression of the three genes were: 7.6, 2.0 and 240.5-fold for kstD1, $k s t D 2$ and $k s t D 3$, respectively, in cultures grown in cholesterol; and 13.6, 0.7 and 0.6-fold for kstD1, kstD2 and $k s t D 3$, respectively, in cultures grown in AD.

The particular organization of $R$. ruber Chol-4 kstD1 and $k s t D 3$ genes (Fig. 1) opens the possibility that polycistronic $k s t D$ mRNAs could be synthesized by the cotranscription of the cyp450-kstD1-MFS transporter and $k s t D 3-h s d 4 B-c h o G$ gene clusters. The results of the RTPCR experiments did not show the occurrence in AD culture medium of either cyp450-kstD1 or kstD1-MFS transporter RNA sequences, indicating that $k s t D 1$ gene is independently transcribed.

In contrast, co-transcripts from both kstD3-hsd $4 B$ ORFs and $h s d 4 B-c h o G$ ORFs could be amplified from cultures grown in the presence of cholesterol, strongly suggesting that kstD3-hsd4B-choG ORFs are co-transcribed into a polycistronic mRNA (Fig. 1). This group of three genes are also described to be co-transcribed in $R$. erythropolis [27].

The adjacent location of kstD3-hsd4B ORFs is highly conserved among rhodococci. The $h s d 4 B$ ORF encodes a 2-enoyl acyl-CoA hydratase involved in the $\beta$-oxidative cycle of the C-17 cholesterol side chain [65]. The choG ORF encodes an extracellular cholesterol oxidase that it is involved in the first step of cholesterol catabolism that implies its conversion to 4-cholesten-3-one [27, 47, 6769]. All this suggests that kstD3, hsd $4 B$ and $c h o G$ genes are mainly involved in the steroid catabolism.

In this work we showed that kstD2 is constitutively transcribed, while $k s t D 1$ and $k s t D 3$ are also constitutively but faintly transcribed, although they can be highly induced by $\mathrm{AD}$ or $\mathrm{CHO}$. In a previous work [24], we reported the construction of a kstD2 deletion mutant of $R$. ruber that is unable to grow in minimal medium supplemented with $\mathrm{AD}$. The question is why KstD1 and/ or KstD3 cannot substitute KstD2 allowing the kstD2 deletion mutant to grow on AD. To partially advance in this subject, RT-PCR experiments were also performed on RNA from the kstD2 $R$. ruber mutant to check the expression of the other $k s t D$ genes (Fig. $5 \mathrm{~b}$ ).
The results showed that the transcription pattern of the $k s t D$ genes of the mutant is far different than that of the same genes in the wild type. Namely, kstD1 gene in the mutant was constitutively and slightly transcribed and its expression is induced in CHO. A more noticeable change affects to the kstD3 gene, which is not transcribed at all in any of the conditions used. These data reveal a complex relationship among the KstD enzymes and their expression control mechanisms. Modification of the $k s t D$ transcription levels of genes remaining in the cell have also been described in a $M$. neoaurum kstD mutant: the transcription ratio of the kstD1 ORF (similar to the $k s t D 3$ ORF from $R$. ruber) in $\mathrm{AD}$ induced cultures respect to glycerol cultures increases from 1.8 (in the wild type strain) to 2.7 -fold (in the kstD3 M. neoaurum mutant strain) [66].

An appealing conclusion of that complex situation is that the three KstD proteins of $R$. ruber strain Chol-4 may be differentially involved in distinct pathways of steroid degradation, and that their expression could also be differentially and specifically controlled.

\section{Heterologous expression of KstD1, KstD2 and KstD3 of $R$. ruber strain Chol-4}

The three $R$. ruber $k s t D$ genes (kstD1, kstD2 and kstD3) were cloned into the pTip-QC1 expression vector. $R$. erythropolis CECT3014 cells were electroporated with these constructions and clones harbouring each of those recombinant plasmids were isolated. Expression of the KstD proteins from these vectors in the CECT3014 transformed cells was followed by SDS-PAGE analysis. Molecular weights were $54.8,60.8$ and $61.8 \mathrm{kDa}$ for KstD1, KstD2 and KstD3 respectively (Additional file 5). Cellfree extracts of cultures grown from these clones were used for the analysis of KstDs activities, and the kinetic parameters of the heterologously expressed KstDs from R. ruber were followed for different substrates (Table 1; see also Additional file 3 for substrates structure). Control cell extract from the R. erythropolis culture harbouring an empty $\mathrm{pTip}-\mathrm{QC} 1$ vector yielded none or very low basal levels when acting on all the substrates used in the assay and were taken into account for final activities.

The substrate profile of KstD1 showed a clear preference to 9OHAD and testosterone, followed by progesterone, Deoxy corticosterone (DOC) and AD (Table 1). All these compounds display a keto group at C3, a C4-C5 double bond and an electronegative side-chain at $\mathrm{C} 17$ (Additional file 3). When comparing to the substrate preference order of $R$. erythropolis SQ1 KstD1 (Prog > 9OHAD, AD > $5 \alpha$-Tes $>$ BNC $>11 \beta$ Cort), some details highlights: (i) KstD1 $1_{\mathrm{SQ} 1}$ has a relative catalytic efficiency (RCE) on progesterone 3.4 times higher than on 


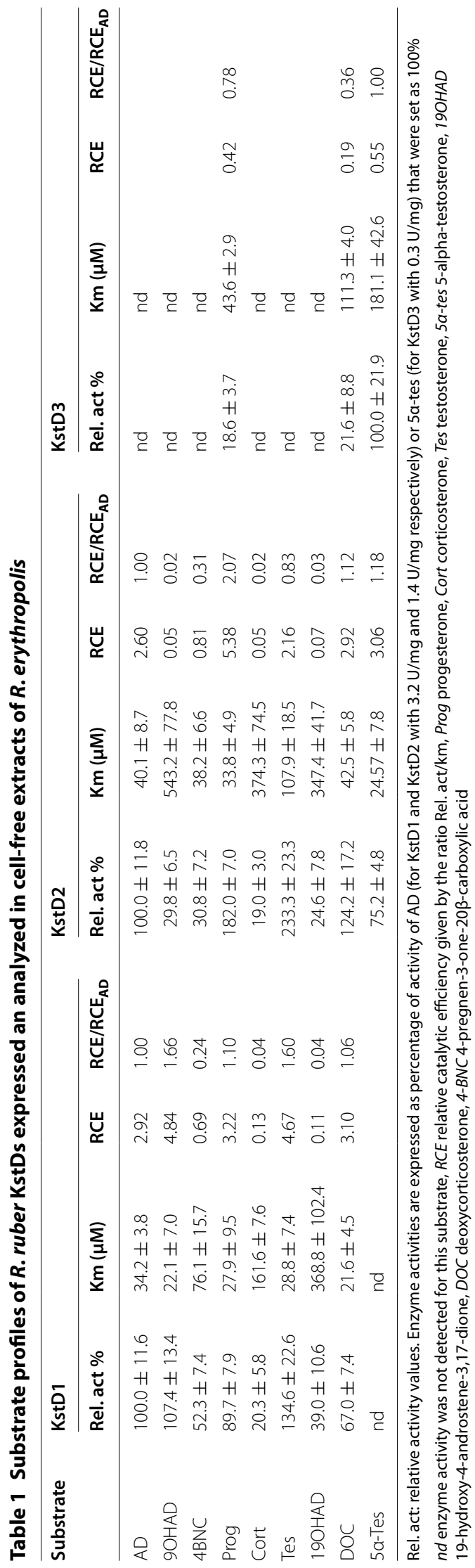


$\mathrm{AD}$, a very big difference to the ratio 9OHAD/AD (1.6) showed by $R$. ruber KstD1 (Table 1); (ii) $R$. ruber KstD1 is not active on $5 \alpha$-Tes in contrast to KstD $1_{\mathrm{SQ} 1}$.

The order of substrate preference of $R$. ruber KstD2 placed progesterone in the first position, followed by $5 \alpha$-Tes, DOC, AD and testosterone (Table 1), displaying a substrate profile similar to $\mathrm{KstD} 2{ }_{\mathrm{SQ}} 1$ enzyme (Prog $>$ AD $>5 \alpha-$ Tes $>$ 9OHAD, BNC $>11 \beta$ Cort). It is noteworthy the similarity of both KstD profiles, having in mind that they have been expressed in different cellular context (a Rhodococcus strain, and E. coli). R. ruber KstD2 has a broader range of substrates than $R$. ruber KstD1 as it can act on all the KstD1 substrates and also on $5 \alpha$-Tes that contains a saturated A ring (Table 1).

Rhodococcus ruber KstD3 did not show any activity at all when acting on $\mathrm{AD}$ or $9 \mathrm{OHAD}$, which are considered the natural substrates for KstD enzymes, but it showed the highest activity when using $5 \alpha$-Tes as substrate followed by progesterone and lastly DOC (Table 1). R. ruber KstD3 has a very narrow substrate range similarly to the R. erythropolis SQ1 and M. tuberculosis $\mathrm{H} 37 \mathrm{Rv}$ isoforms, being the A-ring saturated $5 \alpha$-Tes the preferred substrate for all KstD3 enzymes (Table 1, [20]). However, KstD3 affinity for $5 \alpha$-Tes differs from $33-36 \mu \mathrm{M}$ in the cases of $\mathrm{KstD}_{\mathrm{H} 37 \mathrm{RV}}$ and $\mathrm{KstD} 3_{\mathrm{SQ} 1}$ respectively, to $181 \mu \mathrm{M}$ in $R$. ruber KstD3 (Table 1). Despite this low affinity for $5 \alpha$-Tes, this is the best substrate for the $R$. ruber enzyme among those that were assayed (Table 1 ).

On the other hand, although it has been proposed that only steroids carrying a small or no aliphatic side chain at C-17 are suitable substrates for KstD3 [20], a minor activity, not very different to that obtained with progesterone, has been observed in the assays of $R$. ruber KstD3 enzyme on DOC (Additional file 3; Table 1). $R$. ruber KstD3 seems to be more related to the cholesterol metabolism than to the AD metabolism [24] and then it could be acting on some not yet neatly defined intermediaries of the bacterial cholesterol metabolism.

None of the three $R$. ruber KstD proteins displayed detectable activity on 4-cholestene-3-one, $5 \alpha$-cholestane3 -one or $5 \beta$-androstane-3,17-dione (5 $\beta$-AD), ADD, cholesterol, cholestenone, cholic acid, DHEA, ergosterol, stigmasterol, $\beta$-estradiol, sodium deoxycholate or 5 -pregen-3- $\beta$-nolone. Therefore, these KstDs catalyse preferentially 4-ene-3-oxosteroids.

\section{Molecular modelling of KstD1 of R. ruber strain Chol-4}

The sequence of the three KstDs of $R$. ruber were described previously [24]. In an attempt to go deeper inside the reasons of the catalytic and kinetic differences among some of these enzymes, we performed protein sequence analysis and modelling studies on the $R$. ruber KstDs using different approaches. The published three-dimensional structure and the catalytic mechanism proposed for KstD1 from $R$. erythropolis SQ1 has been used as a suitable model [21, 70]. The I-Tasser and PredictProtein programmes predicted that none of the three $R$. ruber KstDs contain sulphur bridges or transmembrane segments.

The catalytic mechanism of $R$. erythropolis SQ1 KstD1 is based in the keto-enol tautomerization of the substrate caused by Tyr 487 and Gly 491 residues that increases the acidity of the $\mathrm{C} 2$ hydrogen atoms of the substrate. Then Tyr119 and Tyr318 capture the axial $\beta$-hydrogen from $\mathrm{C} 2$ as a proton whereas the FAD molecule accepts the axial $\alpha$-hydrogen from the $\mathrm{C} 1$ atom of the substrate as a hydride ion $[21,70]$. Tables 2 and 3 collects the residues involved in the active site of $\mathrm{KstD} 1_{\mathrm{SQ} 1}$ described for Rohman et al. [21] and the homologue residues of the three KstDs from $R$. ruber found by COBALT

Table 2 Homologous residues of KstDs implicated in the catalytic pocket attending to Rohman et al. [21]

\begin{tabular}{llll}
\hline KstD1-SQ1 & KstD1-ruber & KstD2-ruber & KstD3-ruber \\
\hline S52 & G53 & A59 & G50 \\
F116 & W118 & Y125 & Y116 \\
Y119 & Y121 & Y128 & Y119 \\
F294 & F295 & P337 & F327 \\
V296 & L297 & V339 & S330 \\
Y318 & Y319 & Y366 & Y354 \\
I352 & T356 & & I395 \\
I354 & V358 & F405 & P397 \\
L447 & L447 & L499 & L490 \\
Y487 & Y487 & Y539 & Y530 \\
P490 & G490 & A542 & P533 \\
G491 & G491 & G543 & G534 \\
V492 & N492 & A544 & A535 \\
P493 & P493 & T545 & T537 \\
\hline
\end{tabular}

Italic files contain the four key residues of the KstD1 active site reported for $R$. erythropolis SQ1: Tyr119, Tyr318, Tyr487 and Gly491

Table 3 PredictProtein prediction of the secondary structure and solvent accessibility in \% of the three KstDs from $R$. ruber

\begin{tabular}{lccc}
\hline Structure & KstD1 & KstD2 & KstD3 \\
\hline Strand & 12.72 & 11.88 & 64.74 \\
Loop & 63.99 & 64.01 & 14.38 \\
Helix & 23.29 & 24.11 & 20.88 \\
\hline Accessibility & KstD1 & KstD2 & KstD3 \\
\hline Exposed & 24.1 & 21.81 & 22.11 \\
Buried & 67.7 & 71.63 & 70.5 \\
Intermediate & 8.22 & 6.56 & 7.54 \\
\hline
\end{tabular}


programme. The four key residues: Tyr119, Tyr318, Tyr487 and Gly491 of the KstD1 $1_{\mathrm{SQ} 1}$ active site have a counterpart residue in the $R$. ruber KstDs. However, the I-TASSER model prediction of these $R$. ruber enzymes shows that the orientations of the side chain of the tyrosine residues differ within the catalytic pocket site being specific of each enzyme. The variation in orientation of the key residues inside the catalytic poked is shown in Fig. 6. Particularly, the orientation of KstD3 Y354 and Y530 residues is almost opposite to that of its homologous KstD1 and KstD2 residues. Moreover, amino acid Y128 from KstD2 has a position highly separated compared to its homologous from KstD1 (Y121) and KstD3 (Y119). These differences could justify the different affinity and catalytic properties among the three KstDs. The current shortage of crystalline structures of these proteins greatly limit more detailed conclusions.

A redundancy of KstD and Ksh enzymes have been described in the actinobacteria genomes [20, 24, 64, 70]. This redundancy could provide the cell with a bigger metabolic versatility and a fine-tuned response to the challenging environment. In the case of KstD enzymes, three homologues have been found in R. erythropolis strain SQ1 and in $M$. neoaurum that displayed different substrate preferences and that could be involved in different metabolic steps in a strain-dependent way $[20,32,66]$.

Even more, it has been shown recently that mutations in the KstDs provokes a different ADD/AD molar ratio [71] and that environmental factors such as an increase of temperature can inhibit the KstD/Ksh action [72] on phytosterol in Mycobacterium sp. These multiplicity and versatility give to these enzymes a substantial role in the catalytic dehydrogenation of several related steroid molecules and pose many difficulties to clarify the particular role and way of acting of every single KstD.

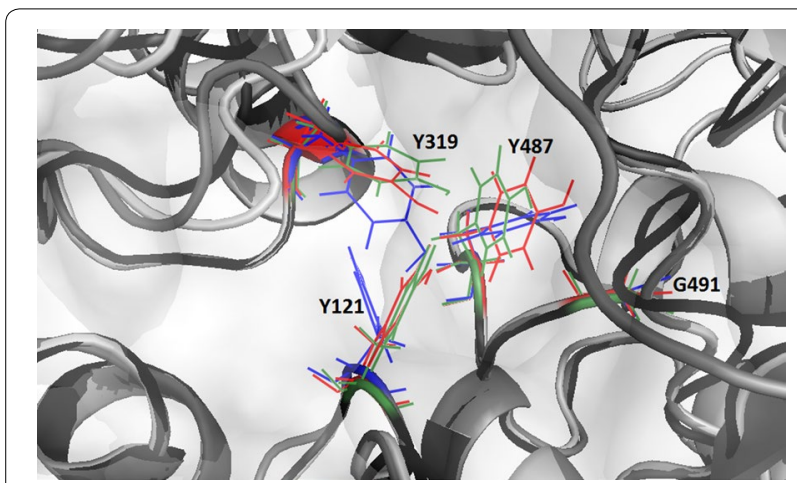

Fig. 6 Modelling of the active site of KstDs. The orientation of the four key residues in the KstD binding pocket is shown. They are superposed and depicted in different colours: green for KstD1, blue for KstD2 and red for KstD3. Only KsD1 residues are named, their counterpart homologues residues of KstD2 and KstD3 being listed in Tables 2 and 3
Our results suggest that both KstD1 and KstD2 of $R$. ruber could act in the conversion of AD to ADD being KstD1 mainly involved in the 9OHAD to 9OHADD conversion, in a similar way to what has been described in R. erythropolis SQ1 [32]. However, there are differences between these two strains, as the necessity of a double kstD1 and kstD2 mutation to prevent the growth in $\mathrm{AD}$ in the case of $R$. erythropolis SQ1 [32] or R. rhodochrous DSM43269 [73], while the same effect is obtained by the single kstD2 deletion in $R$. ruber strain Chol-4 [24], suggesting that this last mutation affects in some way the activity of the KstD1 protein.

$k s t D 3$ ORF occurs in the $R$. ruber genome in a quite conserved location within Rhodococcus species, clustered with $h s d 4 B$ (which encodes a 2-enoyl acyl-CoA hydratase proposed to be involved in cholesterol side-chain shortening) [65] and choG (coding for a cholesterol oxidase that converts cholesterol into cholestenone) [27, 47, 68]. Recently it has been proposed that sterols can be catabolised in $R$. equi USA-18 by two partially different pathways, namely via $\mathrm{AD}$ or via $\triangle 1,4-\mathrm{BNC}$, that converge in the intermediary 9OHADD [74]. Given its substrate preference, the fact that the growth in $\mathrm{AD}$ is independent of the KstD3 activity while the growth in cholesterol needs the presence of either KstD2 or KstD3 in R. ruber [24], lead us to suggest that KstD3 may be involved in an alternative $\mathrm{AD}$-independent cholesterol catabolic pathway.

\section{Conclusions}

To sum up, this study provides biochemical and genetic insights into the three KstD proteins found in $R$. ruber. The kinetic differences between the three KstDs suggest that each enzyme could act on different steps of the steroid catabolic routes. Both KstD1 and KstD2 could be involved in the AD catabolism while KstD1 would have a preference for 9OHAD and KstD2 for progesterone. KstD2 seems to be a more versatile enzyme than KstD1 in $R$. ruber as it can act also on saturated steroid substrates such as $5 \alpha$-Tes. On the other hand, the narrower range of substrates for KstD3 and its preference for saturated steroid made this enzyme different to KstD1 and KstD2 and suggest that it may be involved in AD-independent steroid catabolism. The differences found in the orientation of catalytic residues of each KstD within the binding pocket site could explain the substrates preferences of each enzyme.

The promoter regions that support transcription of $k s t D$ genes have been cloned and functionally identified. The three promoter boxes contain different expression patterns, from the TCCG motif found in the kshA-kstD2 ORF intergenic region to the KstR boxes in the $h s a E$ $k s t D 3$ intergenic region. Moreover, $k s t D 3$ ORF was transcribed as a polycistronic kstD3-hsd4B-choG mRNA and 
was induced in cholesterol growing media, reinforcing the role of KstD3 in the cholesterol metabolism. Potential functions of $R$. ruber strain Chol-4 KstDs in other steroid pathways remain to be elucidated.

\section{Additional files}

Additional file 1. Bacterial strains and plasmids used in this work.

Additional file 2. Primers used in this work. Restriction sites are marked in bold.

Additional file 3. Structure of the steroids used in this work.

Additional file 4. Scheme of the pNVSP vectors construction. The shuttle vector pNV119 was modified to include a mcs from pSEVA351 (pNVS) and after that coupled with the apramycin resistance ORF. Intergenic regions containing the putative $k s t D$ promoters were cloned in the mcs to obtain pNVSP vectors.

Additional file 5. Expression of KstDs from ptip-QC1 vectors in induced R. erythropolis CECT3014 cells. SDS-PAGE analysis on a $12.5 \%$ gel was performed using $10 \mu \mathrm{g}$ of the cell-free extracts. The band corresponding to KstD overexpression is marked with a rectangle. Precision plus protein standard from Bio-Rad was used as size marker.

Additional file 6. R. ruber strain Chol-4 ORF4 and ORF5 protein sequences.

\section{Abbreviations}

AD: 4-androstene-3,17-dione; ADD: 1,4-androstadiene-3,17-dione; 5B-AD: 5 $\beta$-androstane-3,17-dione; 9OHAD: 9a-hydroxy-4-androstene-3,17-dione; 9OHADD: 9a-hydroxy-1,4-androstadiene-3,17-dione; 4-BNC: 4-pregnen-3-one20ß-carboxylic acid; 5 a-Tes: $17 \beta$-hydroxy-5a-androstan-3-one; DOC: deoxy corticosterone; ORF: open reading frame; DCPIP: 2,6-dichlorophenol-indophenol; KstD: 3-ketosteroid- $\triangle^{1}$-dehydrogenase; LB: Luria-Bertani; mcs: multiple cloning site; TSS: transcriptional start site.

\section{Authors' contributions}

GG made the heterologous expression in R. erythropolis, the coexpression study, the RT-qPCR and the promoter characterization. JMNLL and GG performed the bioinformatic analyses. LFH carried out the RT-PCR experiment and collaborated with GG in the heterologous expression experiments. JP and JMNLL drafted the manuscript. JP, JMNLL and GG participated in the design and coordination of the study. All authors read and approved the final manuscript.

\section{Author details}

${ }^{1}$ Department of Biochemistry and Molecular Biology I, Universidad Complutense de Madrid, 28040 Madrid, Spain. ${ }^{2}$ Faculty of Science and Engineering, Microbial Physiology-Gron Inst Biomolecular Sciences \& Biotechnology, Nijenborgh 7, 9747 AG Groningen, The Netherlands.

\section{Acknowledgements}

We are in debt to R. van der Geize (Netherland) and to the group of J. L. García (CIB, CSIC) for their scientific support and invaluable help. We thank Rodrigo Velasco for his help on molecular modelling and Sara Montero for her help on kinetics.

\section{Competing interests}

The authors declare that they have no competing interests.

\section{Availability of data and materials}

The datasets supporting the conclusions of this article are included within the article and its supplementary materials.

\section{Funding}

This work was funded by projects BFU2009-11545-C03-02, BIO201239695-CO2-01 and RTC-2014-2249-1 (GG is under contract of this last project) from the Spanish Ministry of Education and Science.
Received: 8 August 2016 Accepted: 7 March 2017

Published online: 14 March 2017

\section{References}

1. Iwabuchi N, Sunairi M, Urai M, Itoh C, Anzai H, Nakajima M, Harayama S. Extracellular polysaccharides of Rhodococcus rhodochrous S-2 stimulate the degradation of aromatic components in crude oil by indigenous marine bacteria. Appl Environ Microbiol. 2002;68:2337-43.

2. McLeod MP, Warren RL, Hsiao WW, Araki N, Myhre M, Fernandes C, Miyazawa D, Wong W, Lillquist AL, Wang D, et al. The complete genome of Rhodococcus sp. RHA1 provides insights into a catabolic powerhouse. Proc Natl Acad Sci USA. 2006;103:15582-7.

3. Yam KC, Okamoto S. Adventures in Rhodococcus - from steroids to explosives. Can J Microbiol. 2011;57:155-68.

4. Barel-Cohen K, Shore LS, Shemesh M, Wenzel A, Mueller J, Kronfeld-Schor N. Monitoring of natural and synthetic hormones in a polluted river. J Environ Manag. 2006;78:16-23.

5. Gracia T, Jones PD, Higley EB, Hilscherova K, Newsted JL, Murphy MB, Chan AK, Zhang X, Hecker M, Lam PK, et al. Modulation of steroidogenesis by coastal waters and sewage effluents of Hong Kong, China, using the H295R assay. Environ Sci Pollut Res Int. 2008;15:332-43.

6. Soto AM, Calabro JM, Prechtl NV, Yau AY, Orlando EF, Daxenberger A, Kolok AS, Guillette LJ Jr, le Bizec B, Lange IG, et al. Androgenic and estrogenic activity in water bodies receiving cattle feedlot effluent in Eastern Nebraska, USA. Environ Health Perspect. 2004;112:346-52.

7. van der Geize R, Dijkhuizen L. Harnessing the catabolic diversity of rhodococci for environmental and biotechnological applications. Curr Opin Microbiol. 2004;7:255-61.

8. Malaviya A, Gomes J. Androstenedione production by biotransformation of phytosterols. Bioresour Technol. 2008;99:6725-37.

9. García JL, Uhía I, Galan B. Catabolism and biotechnological applications of cholesterol degrading bacteria. Microb Biotechnol. 2012;5:679-99.

10. Horinouchi M, Hayashi T, Kudo T. Steroid degradation in Comamonas testosteroni. J Steroid Biochem Mol Biol. 2012;129:4-14.

11. Philipp B. Bacterial degradation of bile salts. Appl Microbiol Biotechnol. 2011;89:903-15.

12. Uhía I, Galán B, Kendall SL, Stoker NG, García JL. Cholesterol metabolism in Mycobacterium smegmatis. Environ Microbiol Rep. 2012;4:168-82.

13. Petrusma M, van der Geize R, Dijkhuizen L. 3-Ketosteroid 9alpha-hydroxylase enzymes: rieske non-heme monooxygenases essential for bacterial steroid degradation. Antonie Van Leeuwenhoek. 2014;106:157-72.

14. Florin C, Kohler T, Grandguillot M, Plesiat P. Comamonas testosteroni 3-ketosteroid-delta 4(5 alpha)-dehydrogenase: gene and protein characterization. J Bacteriol. 1996;178:3322-30.

15. Itagaki E, Hatta T, Wakabayashi T, Suzuki K. Spectral properties of 3-ketosteroid-delta 1-dehydrogenase from Nocardia corallina. Biochim Biophys Acta. 1990;1040:281-6.

16. Itagaki E, Wakabayashi T, Hatta T. Purification and characterization of 3-ketosteroid-delta 1-dehydrogenase from Nocardia corallina. Biochim Biophys Acta. 1990;1038:60-7.

17. Chen MM, Wang FQ, Lin LC, Yao K, Wei DZ. Characterization and application of fusidane antibiotic biosynthesis enzyme 3-ketosteroid1-dehydrogenase in steroid transformation. Appl Microbiol Biotechnol. 2012;96:133-42.

18. Kisiela M, Skarka A, Ebert B, Maser E. Hydroxysteroid dehydrogenases (HSDs) in bacteria - a bioinformatic perspective. J Steroid Biochem Mol Biol. 2012;129:31-46.

19. Hilyard EJ, Jones-Meehan JM, Spargo BJ, Hill RT. Enrichment, isolation, and phylogenetic identification of polycyclic aromatic hydrocarbondegrading bacteria from Elizabeth River sediments. Appl Environ Microbiol. 2008;74:1176-82.

20. Knol J, Bodewits K, Hessels Gl, Dijkhuizen L, van der Geize R. 3-Keto5alpha-steroid Delta(1)-dehydrogenase from Rhodococcus erythropolis SQ1 and its orthologue in Mycobacterium tuberculosis H37Rv are highly specific enzymes that function in cholesterol catabolism. Biochem J. 2008:410:339-46 
21. Rohman A, van Oosterwijk N, Thunnissen AM, Dijkstra BW. Crystal structure and site-directed mutagenesis of 3-ketosteroid Delta1-dehydrogenase from Rhodococcus erythropolis SQ1 explain its catalytic mechanism. J Biol Chem. 2013;288:35559-68.

22. Molnár I, Choi KP, Yamashita M, Murooka Y. Molecular cloning, expression in Streptomyces lividans, and analysis of a gene cluster from Arthrobacter simplex encoding 3-ketosteroid-delta 1-dehydrogenase, 3-ketosteroiddelta 5-isomerase and a hypothetical regulatory protein. Mol Microbiol. 1995;15:895-905.

23. Wierenga RK, Terpstra P, Hol WG. Prediction of the occurrence of the ADPbinding beta alpha beta-fold in proteins, using an amino acid sequence fingerprint. J Mol Biol. 1986;187:101-7.

24. de las Heras LF, van der Geize R, Drzyzga O, Perera J, Navarro Llorens JM. Molecular characterization of three 3-ketosteroid- $\Delta^{1}$-dehydrogenase isoenzymes of Rhodococcus ruber strain Chol-4. J Steroid Biochem Mol Biol. 2012;132:271-81.

25. Fernández de las Heras L, García Fernández E, Navarro Llorens JM, Perera J, Drzyzga O. Morphological, physiological, and molecular characterization of a newly isolated steroid-degrading actinomycete, identified as Rhodococcus ruber strain Chol-4. Curr Microbiol. 2009;59:548-53.

26. Klein U, Gimpl G, Fahrenholz F. Alteration of the myometrial plasma membrane cholesterol content with b-cyclodextrin modulates the binding affinity of the oxytocin receptor. Biochemistry. 1995;34:13784-93.

27. Fernández de las Heras L, Mascaraque V, García Fernández E, NavarroLlorens JM, Perera J, Drzyzga O. ChoG is the main inducible extracellular cholesterol oxidase of Rhodococcus sp. strain CECT3014. Microbiol Res. 2011;166:403-18.

28. Sambrook J, Fritsch EF, Maniatis T. Molecular cloning: a laboratory manual. New York: Cold Spring Harbor Laboratory Press; 1989.

29. Hosek J, Svastova P, Moravkova M, Pavlik I, Bartos M. Methods of mycobacterial DNA isolation from different biological material: a review. Vet Res. 2006;524:180-92.

30. Nakashima N, Tamura T. Isolation and characterization of a rolling-circletype plasmid from Rhodococcus erythropolis and application of the plasmid to multiple-recombinant-protein expression. Appl Environ Microbiol. 2004;70:5557-68.

31. Bradford MM. A rapid and sensitive method for the quantitation of microgram quantities of protein utilizing the principle of protein-dye binding. Anal Biochem. 1976;72:248-54.

32. van der Geize R, Hessels Gl, Dijkhuizen L. Molecular and functional characterization of the kstD2 gene of Rhodococcus erythropolis SQ1 encoding a second 3-ketosteroid $\Delta^{1}$-dehydrogenase isoenzyme. Microbiology. 2002;148:3285-92.

33. de las Heras LF, Alonso S, de Leon AD, Xavier D, Perera J, Navarro Llorens JM. Draft genome sequence of the steroid degrader Rhodococcus ruber strain Chol-4. Genome Announc. 2013. doi:10.1128/ genomeA.00215-13.

34. Petersen TN, Brunak S, von Heijne G, von Nielsen H. SignalP 4.0: discriminating signal peptides from transmembrane regions. Nat Methods. 2011:8:785-6.

35. Solovyev V, Salamov A. Automatic annotation of microbial genomes and metagenomic sequences. In: Li RW, editor. Metagenomics and its applications in agriculture, biomedicine and environmental studies. New York: Nova Science Publishers; 2011. p. 61-78.

36. Reese MG. Application of a time-delay neural network to promoter annotation in the Drosophila melanogaster genome. Comput Chem. 2001:26:51-6.

37. de Jong A, Pietersma H, Cordes M, Kuipers OP, Kok J. PePPER: a webserver for prediction of prokaryote promoter elements and regulons. BMC Genom. 2012;13:299.

38. Yang J, Yan R, Roy A, Xu D, Poisson J, Zhang Y. The I-TASSER Suite: protein structure and function prediction. Nat Methods. 2015;12:7-8.

39. Yachdav G, Kloppmann E, Kajan L, Hecht M, Goldberg T, Hamp T, Honigschmid P, Schafferhans A, Roos M, Bernhofer M, et al. PredictProtein-an open resource for online prediction of protein structural and functional features. Nucl Acids Res. 2014;42:W337-43.

40. Papadopoulos JS, Agarwala R. COBALT: constraint-based alignment tool for multiple protein sequences. Bioinformatics. 2007;23:1073-9.

41. Durante-Rodriguez G, de Lorenzo V, Martinez-Garcia E. The Standard European Vector Architecture (SEVA) plasmid toolkit. Methods Mol Biol. 2014;1149:469-78.
42. Silva-Rocha R, Martínez-García E, Calles B, Chavarría M, Arce-Rodríguez A, de Las Heras A, Paez-Espino AD, Durante-Rodríguez G, Kim J, Nikel $\mathrm{PI}$, et al. The Standard European Vector Architecture (SEVA): a coherent platform for the analysis and deployment of complex prokaryotic phenotypes. Nucl Acids Res. 2013;41:D666-75.

43. Chiba K, Hoshino Y, Ishino K, Kogure T, Mikami Y, Uehara Y, Ishikawa J. Construction of a pair of practical Nocardia-Escherichia coli shuttle vectors. Jpn J Infect Dis. 2007;60:45-7.

44. Gust B, Challis GL, Fowler K, Kieser T, Chater KF. PCR-targeted Streptomyces gene replacement identifies a protein domain needed for biosynthesis of the sesquiterpene soil odor geosmin. Proc Natl Acad Sci USA. 2003;100:1541-6.

45. Hopwood DA, Bibb MJ, Chater KF, Kieser T, Bruton CJ, Kieser HM, Lydiate DJ, Smith CP, Ward JM, Schrempf H. Genetic manipulation of Streptomyces. Norwich: The John Innes Foundation, Cold Spring Harbour Laboratory; 1985

46. Wang C, Lee J, Deng Y, Tao F, Zhang LH. ARF-TSS: an alternative method for identification of transcription start site in bacteria. Biotechniques. 2012. doi:10.2144/000113858.

47. de las Heras LF, Perera J, Navarro Llorens JM. Cholesterol to cholestenone oxidation by ChoG, the main extracellular cholesterol oxidase of Rhodococcus ruber strain Chol-4. J Steroid Biochem Mol Biol. 2014:139:33-44.

48. Kendall SL, Withers M, Soffair CN, Moreland NJ, Gurcha S, Sidders B, Frita R, Ten Bokum A, Besra GS, Lott JS, et al. A highly conserved transcriptional repressor controls a large regulon involved in lipid degradation in Mycobacterium smegmatis and Mycobacterium tuberculosis. Mol Microbiol. 2007:65:684-99.

49. Crowe AM, Stogios PJ, Casabon I, Evdokimova E, Savchenko A, Eltis LD. Structural and functional characterization of a ketosteroid transcriptional regulator of Mycobacterium tuberculosis. J Biol Chem. 2015;290:872-82.

50. Uhía I, Galán B, Medrano FJ, García JL. Characterization of the KstRdependent promoter of the first step of cholesterol degradative pathway in Mycobacterium smegmatis. Microbiology. 2011;157:2670-80.

51. García-Fernández E, Medrano FJ, Galán B, García JL. Deciphering the transcriptional regulation of cholesterol catabolic pathway in mycobacteria: identification of the inducer of KstR repressor. J Biol Chem. 2014;289:17576-88.

52. Ho NA, Dawes SS, Crowe AM, Casabon I, Gao C, Kendall SL, Baker EN, Eltis $L D$, Lott JS. The structure of the transcriptional repressor KstR in complex with $\mathrm{CoA}$ thioester cholesterol metabolites sheds light on the regulation of cholesterol catabolism in Mycobacterium tuberculosis. J Biol Chem. 2016;291:7256-66.

53. Shtratnikova VY, Schelkunov MI, Fokina V, Pekov YA, Ivashina T, Donova MV. Genome-wide bioinformatics analysis of steroid metabolismassociated genes in Nocardioides simplex VKM Ac-2033D. Curr Genet. 2016;62:643-56.

54. Ma Y, Yu H. Engineering of Rhodococcus cell catalysts for tolerance improvement by sigma factor mutation and active plasmid partition. J Ind Microbiol Biotechnol. 2012:39:1421-30.

55. Bashyam MD, Kaushal D, Dasgupta SK, Tyagi AK. A study of mycobacterial transcriptional apparatus: identification of novel features in promoter elements. J Bacteriol. 1996; 178:4847-53.

56. Kendall SL, Burgess P, Balhana R, Withers M, Ten Bokum A, Lott JS, Gao C, Uhia-Castro I, Stoker NG. Cholesterol utilization in mycobacteria is controlled by two TetR-type transcriptional regulators: kstR and kstR2. Microbiology. 2010;156:1362-71.

57. Shell SS, Wang J, Lapierre P, Mir M, Chase MR, Pyle MM, Gawande R, Ahmad R, Sarracino DA, loerger TR, et al. Leaderless transcripts and small proteins are common features of the mycobacterial translational landscape. PLoS Genet. 2015;11:e1005641.

58. StrohI WR. Compilation and analysis of DNA sequences associated with apparent streptomycete promoters. Nucleic Acids Res. 1992;20:961-74.

59. Pfleiderer C, Smid A, Bartsch I, Grummt I. An undecamer DNA sequence directs termination of human ribosomal gene transcription. Nucleic Acids Res. 1990;18:4727-36.

60. Grummt I, Kuhn A, Bartsch I, Rosenbauer H. A transcription terminator located upstream of the mouse rDNA initiation site affects rRNA synthesis. Cell. 1986;47:901-11.

61. Henderson S, Sollner-Webb B. A transcriptional terminator is a novel element of the promoter of the mouse ribosomal RNA gene. Cell. 1986;47:891-900. 
62. Henderson SL, Ryan K, Sollner-Webb B. The promoter-proximal rDNA terminator augments initiation by preventing disruption of the stable transcription complex caused by polymerase read-in. Genes Dev. 1989;3:212-23.

63. Szoköl J, Rucká L, Simciková M, Halada P, Nesvera J, Pátek M. Induction and carbon catabolite repression of phenol degradation genes in Rhodococcus erythropolis and Rhodococcus jostii. Appl Microbiol Biotechnol. 2014;98:8267-79.

64. Mathieu JM, Mohn WW, Eltis LD, LeBlanc JC, Stewart GR, Dresen C, Okamoto K, Alvarez PJ. 7-ketocholesterol catabolism by Rhodococcus jostii RHA1. Appl Environ Microbiol. 2010;76:352-5.

65. van der Geize R, Yam K, Heuser T, Wilbrink MH, Hara H, Anderton MC, Sim E, Dijkhuizen L, Davies JE, Mohn WW, et al. A gene cluster encoding cholesterol catabolism in a soil actinomycete provides insight into Mycobacterium tuberculosis survival in macrophages. Proc Natl Acad Sci USA. 2007;104:1947-52.

66. Yao K, Xu LQ, Wang FQ, Wei DZ. Characterization and engineering of 3-ketosteroid- big up tri, open1-dehydrogenase and 3-ketosteroid9alpha-hydroxylase in Mycobacterium neoaurum ATCC 25795 to produce 9alpha-hydroxy-4-androstene-3,17-dione through the catabolism of sterols. Metab Eng. 2014;24:181-91.

67. Kreit J, Sampson NS. Cholesterol oxidase: physiological functions. FEBS J. 2009;276:6844-56.

68. Pollegioni L, Piubelli L, Molla G. Cholesterol oxidase: biotechnological applications. FEBS J. 2009;276:6857-70.
69. Vrielink A, Ghisla S. Cholesterol oxidase: biochemistry and structural features. FEBS J. 2009;276:6826-43.

70. Petrusma M, Hessels G, Dijkhuizen L, van der Geize R. Multiplicity of 3-ketosteroid-9a-hydroxylase enzymes in Rhodococcus rhodochrous DSM43269 for specific degradation of different classes of steroids. J Bacteriol. 2011;193:3931-40

71. Shao M, Zhang X, Rao Z, Xu M, Yang T, Li H, Xu Z, Yang S. A mutant form of 3-ketosteroid- $\Delta^{1}$-dehydrogenase gives altered androst-1,4-diene-3, 17-dione/androst-4-ene-3,17-dione molar ratios in steroid biotransformations by Mycobacterium neoaurum ST-095. J Ind Microbiol Biotechnol. 2016:43:691-701.

72. Xu XW, Gao XQ, Feng JX, Wang XD, Wei DZ. Influence of temperature on nucleus degradation of 4-androstene-3, 17-dione in phytosterol biotransformation by Mycobacterium sp. Lett Appl Microbiol. 2015;61:63-8.

73. Liu Y, Shen Y, Qiao Y, Su L, Li C, Wang M. The effect of 3-ketosteroid- $\Delta^{1}$ dehydrogenase isoenzymes on the transformation of AD to 9alpha-OHAD by Rhodococcus rhodochrous DSM43269. J Ind Microbiol Biotechnol. 2016:43:1303-11.

74. Yeh CH, Kuo YS, Chang CM, Liu WH, Sheu ML, Meng M. Deletion of the gene encoding the reductase component of 3-ketosteroid 9a-hydroxylase in Rhodococcus equi USA-18 disrupts sterol catabolism, leading to the accumulation of 3-oxo-23,24-bisnorchola-1,4-dien-22-oic acid and 1,4-androstadiene-3,17-dione. Microb Cell Fact. 2014;13:130.

\section{Submit your next manuscript to BioMed Central and we will help you at every step:}

- We accept pre-submission inquiries

- Our selector tool helps you to find the most relevant journal

- We provide round the clock customer support

- Convenient online submission

- Thorough peer review

- Inclusion in PubMed and all major indexing services

- Maximum visibility for your research

Submit your manuscript at www.biomedcentral.com/submit 